\title{
Arc erosion on W plasma facing components in ASDEX Upgrade
}

\author{
V. Rohde* , M. Balden, N. Endstrasser, U.v. Toussaint, and ASDEX Upgrade Team \\ Max-Planck-Institut für Plasmaphysik, EURATOM Association, D-85748 Garching, Germany.
}

\begin{abstract}
:
The occurance of arc traces on plasma phasing components show a strong local variation. Strong erosion is observed at the inner baffle region, where arcing is more dominat than physical sputtering and in total $2 \mathrm{~g}$ of tungsten are eroded during one campaign. On the other hand this is about a factor of ten less than the net physical sputtering in the outer divertor region. Droplet production by arcing is a direct dust source, which covers $15 \%$ of the eroded tungsten. The penetration of these droplets, which have a typical diametre of $2 \mu \mathrm{m}$, into the core plasma is still unclear. Laboratory measurements of the light emission by arcing using a fast camera confirme former data, which show that arcs are active during ELMs.
\end{abstract}

PACS: $52.40 \mathrm{Hf}, 52.25 \mathrm{Xz}$

JNM keywords: First Wall Materials, Plasma Material Interaction, Tungsten PSI-20 Keywords: ASDEX Upgrade, Arcing, Erosion \& Redeposition, First Wall, Tungsten

* Corresponding author address: Max-Planck-Institut für Plasmaphysik, Boltzmannstr. 2, 85478 Garching, Germany

*Corresponding author e-mail: Volker.Rohde@ipp.mpg.de

Presenting author: Volker Rohde

Presenting author e-mail: : Volker.Rohde@ipp.mpg.de 


\section{Introduction}

The transition from carbon-based to high- $\mathrm{Z}$ wall materials reduced strongly the erosion of plasma facing components (PFCs) in ASDEX Upgrade (AUG) [1]. Whereas physical and chemical sputtering dominate for carbon PFCs, other processes as erosion by arcs can play a significant role for high-Z materials.

Whereas erosion by sputter processes is usually not visible by eye, most arc traces could be easily detected. A simple inspection of a fusion device will always find some traces, which could be interpreted as remnants from arcing (Fig 1). Especially at deposition dominated regions, these traces are easily visible, as the colour of the deposited material and the substrate are different [2].

Two kinds of arcs have to be distinguished: arcs at the surface directly in contact with the plasma and arcs at the structures not directly exposed to plasma [3]. Here we deal only with arc traces on PFC surfaces, which are oriented perpendicular to the local magnetic field direction, i.e., which burn and move during periods with the magnetic field on, i.e. plasma discharges.

Whereas a database on the erosion by arcing for different bulk materials exists, the properties of the $\mathrm{W}$ coated $\mathrm{C}$ tiles, which are used in AUG, have been investigated in recent laboratory experiments [4]. 


\section{Erosion of PFCs by arcing}

The amount of arc traces varies strongly at different locations inside the vessel of AUG. Although optical inspections show no strong variation of the amount of arc traces toroidally. Several poloidal positions with increased arcing could be identified. As indicated in Fig 1, four distinct regions, which are highly affected by arcing, exist: upper divertor close to the second X-point (1), upper outer divertor (2), lower inner divertor baffle region (3) and the divertor roof baffle (4). The pictures show that the arc traces may vary even on a centimetre scale on one tile in the poloidal direction (Fig 1b) and toroidal direction (Fig 1c). To estimate the total erosion by arcing, information on the whole PFC surfaces is required, which means that a complete $2 \mathrm{~d}$ scan is needed. In contrast to physical sputtering the erosion by arcing is quite local. Only the locations directly affected by the arcs are eroded, whereas the PFCs in between the arc traces are not affected or even covered by deposited material released by the arcing. As the arc traces have typical widths of 10 to $50 \mu \mathrm{m}$, high resolution measurements are needed. In this paper we used a multi diagnostic approach: first a subset of tiles removed from the vessel were scanned to obtain high resolution pictures. A numerical analysis was applied to get the percentage of the surface affected by arcing. For estimation of the amount of eroded material, the depth of the traces is was measured using confocal laser microscopy with integrated profilometer. To verify the erosion, additionally a scanning electron microscopy (SEM) and a focused ion beam for cutting cross-sectioning were used.

The PFCs in the main chamber of AUG consist of graphite tiles with a 3-10 $\mu$ m thick tungsten coating and deposition layers, typically a:BC-H layers with low surface conductivity. In total 96 tiles were selected and scanned with typically 15000 by 20000 pixels with 48 bit per pixel. For analysis the image is segmented in sub images with roughly 1000 
by 800 pixels. For each sub image and color channel individually a 2 -D linear trend removal is performed to compensate for the large scale illumination conditions. In the subsequent step the probability for a pixel to be part of an arc-track is evaluated. Approaches based on simple intensity thresholds failed because arc-tracks can be either brighter or darker than their surroundings. A common arc track feature, however, is its very elongated, although the width and total length varies considerably. Unfortunately algorithms based on 2-D-FFT or ridgelets based approaches were also not successful, in part because the machined tile surface also exhibited grinding structures very similar in size to the arc tracks. Laborious visual inspection finally revealed that the variance of the color intensity within a track differs from that of a the undamaged surface: the variance is typically lower (the interior of the arc-track appears to be smoother) than its surrounding. Based on that insight the detection algorithm assigns to each pixel the vector of the variances of rectangular boxes of 41 pixel times 3 pixels with the orientation varied between \pm 30 degrees. The resulting histogram is then normalized by the average variance of the sub image to compensate for differences resulting from different illumination conditions. Additionally the normalized second derivative perpendicular to the direction of the elongation with respect to the intensity and the variance is computed for every pixel: arcs should display a typical high-low-high-pattern in variance and an alternating pattern in the intensity. Based on the resulting distributions (arcs result in a deviation - i.e. the occurrence of heavy tails with a characteristic 'knee' - from the normal distribution) individual thresholds are imposed. Pixels were only identified as 'arc-pixels' when all criteria (intensity, variance, second-derivative) hold. In a final step a running edge preserving median filter (also 41 by 3 pixels) is applied to the 2-D arrays computed from the variance and second derivative data to remove individual outliers. 
The performance of this algorithm has been validated using artificial random image data, manually selected arc-free tiles and arc-covered tiles. The false positive level is around $2 \%$, the positive-false level is much harder to assess, but is typically in the same range. Confidence in the robustness of the algorithm is further strengthened by the fact that arc tracks are reliably traced between the individual sub images which are processed fully independently.

To obtain the total erosion, the depth of the traces was measured using confocal laser scanning microscopy. Obviously, this technique could be only applied to small areas, so both methods have to be combined to get the total erosion in AUG. The erosion induced by arcs varies significantly, even at similar locations. Some of the traces can be identified optically, but no significant depth is measured by the profilometer. Strong erosion by arcing is mostly found at deposition dominated regions.

The results of an analysis are visualized in Fig. 2. The fraction of arc traces with respect to the total surface on this tile, removed from the inner divertor baffle region, varies from almost no erosion at the upper left side to $11 \%$ at the right side. On the average $3.3 \%$ of the surface are damaged by arcing on this tile. In reality the fraction of arc damage is even higher, as the algorithm did not detect small holes produced by arcing and the threshold for arc detection was adjusted for safe detection, i.e. some traces are not marked. In addition there are deposited layers on the tile, which can cover arc traces.

Results are compiled in Fig. 3: All tiles of one section of AUG, starting from the top divertor along the central column to the lower divertor, are presented as squares. At the left side the shape of the tiles, as shown in Fig 1 are reploted. Depending on the power flux to the tiles the shape is adjusted to match the magnetic field lines. For this reason up to 8 tiles are used at the divertor and only 2 at the central column. Tiles indicated as grey have not been analyzed 
up to now, other colors show the fraction of arc traces of the total surface. The strongest erosion was detected at the inner baffle region, were up to $6 \%$ of a tile was damaged. All tiles located in this region show strong erosion, but there are significant differences of a factor of two at this section even for the same poloidal location. At the inner divertor always 3 tiles are mounted at the same substructure. This sub pattern is obviously reflected in the arc activity.

Almost no arcing was detected at the central column and the inner divertor strike point module. The roof baffle shows strong erosion by arcing, whereas in the outer divertor only the upper baffle region is affected. There are some tiles, which show significantly more arc traces than the surrounding ones (Bgr 9b-tile 6 and Bgr 2-tile 5). The reason for this local enhancement is not clear.

The typical erosion depth for the different tiles was used to calculate the total amount of eroded tungsten. The results are shown at the right side of Fig. 3 always summed up for one row of tiles. As expected from inspection by eye, the largest tungsten erosion was measured at the inner baffle. Summing up all material, about $1100 \mathrm{mg}$ of tungsten was eroded by arcing. Additional the upper divertor region has to be taken into account. A total erosion of 2 $\mathrm{g}$ of tungsten was estimated from this investigation. This has to be compared with the tungsten erosion by physical sputtering as measured by marker tiles [5]. Strong net erosion is only observed at the outer divertor strike point module, where physical sputtering by impurity atoms dominates. Extrapolating these results to the experimental time of the 2009 campaign, a total net erosion of $24 \mathrm{~g}$ of tungsten is expected. Obviously the tungsten erosion by arcing is about $10 \%$ of the physical sputtering and can be neglected for the global tungsten erosion balance in AUG. However, locally the erosion by arcing can clearly dominate the physical sputtering [2]. 


\section{Dust production by arcing}

In contrast to physical sputtering not only neutrals and ions are released by arcing but also droplets and clusters are produced. In a fusion device the dense plasma in front of the tiles will ionizise the neutrals immediatly, so the neutral fraction will mostly behave as the ions. Droplets are produced by molten material, which forms small spheres consisting mostly of $\mathrm{W}$. The behaviour of droplets is significantly different to sputtered particles, as droplets allow for a long range transport and may have a higher penetration probability into the core plasma. The production of droplets is confirmed by investigation of deposited layers close to the arc traces [6]. In deposition dominated regions the PFCs are covered by additional layers, which will fractionate in the vicinity of the arcs.

Dust investigations using Si wafers as dust collectors have been performed in AUG during the recent years. An automized SEM apparatus allowed analysing some thousand of dust particles [7]. This huge number allows statistically relevant classification and extrapolation of the dust particles observed. Significant amounts of tungsten are observed in two classes: small tungsten spheres and flakes, which consist out of tungsten particles imbedded in a matrix of boron and carbon [8]. The $\mathrm{W}$ spheres are intepreted as droplets released by arcing, whereas the morphology of the flakes hint to the production as deposited layers which are fractionised by arcing. Up to 4 collectors mounted at the same poloidal but different toroidal positions yield almost the same amount of tungsten spheres. Knowledge of the total amount of tungsten spheres requires an extrapolation from the local measurements to the whole vessel. This kind of dust collectors could be only used at positions, where the heat flux is low enough not to influence the Si wafer. As another dust collector mounted at the high field side 
shows almost the same amount of $\mathrm{W}$ spheres it is plausible to assume a homogeneous flux of tungsten spheres in the whole vessel. Additionally almost the same flux of dust particles was observed at a probe exposed using the divertor manipulator system [9]. The investigations yield for the 2009 campaign a flux of 1 tungsten droplet and 9.5 flakes per second and square centimetre. The spheres, consisting of pure $\mathrm{W}$, have a typical diametre of $2.2 \mu \mathrm{m}$ with a standard deviation of $1.6 \mu \mathrm{m}$. The tungsten flakes have a irregular shape and consist of a boron and carbon matrix with small tungsten spheres included. The typical diametre is 0.6 $\mu \mathrm{m}$ with a quite broad distribution of $2.3 \mu \mathrm{m}$. To estimate the total amount of particles produced, a uniform distribution of the particles on the whole toroidal cross section is assumed. The integral on the spheres yields a sum of $267 \mathrm{mg}$ tungsten. Even as the total number of flakes is much higher the smaller diametre and the less tungsten content yields only $26 \mathrm{mg}$ of tungsten. Therefore, in total $15 \%$ of the tungsten eroded by arcs is found as dust particles.

Tungsten eroded as droplets may have a higher probability to penetrate the core plasma, as massive particles may survive the SOL plasma. As known from pellet investigations the velocity of the particle is the key parameter for the penetration. Again laboratory investigations were used to estimate the velocity of the droplets [4]. A two component velocity distribution function with the fast component of $80 \mathrm{~m} / \mathrm{s}$ and a slow one of $20 \mathrm{~m} / \mathrm{s}$ was found. Tungsten particles of some micron diametre and a velocity of some $100 \mathrm{~m} / \mathrm{s}$ are expected to be able to reach the core plasma, but numerical calculations are required to verify this assumption. Low velocity pellets are disturbed by electrical fields at the plasma edge and ejected material from the pellets. Further calculations are needed to clarify this aspect. 


\section{Direct observation of arcs}

Former investigations on arcing suggested that the onset of arcs is mostly during unstable plasma phases: arcing was mostly observed during plasma ramp up and ramp down, but not during the flat top phase [10]. Whereas the current induced by an arc in a tile is smeared by the thermo current, fast cameras can be used to discrimenate the light emission by the arc from the background plasma radiation. In a magnetic field the arc should move perpendicular to the field direction. A fast CMOS camera (26845 fps) was installed at AUG which allows to view onto the inner baffle region with a high spatial resolution of $160 \mu \mathrm{m} /$ pixel [3].

Indeed single light events, which look like arcing are observed. A statistical evalution shows a strong correlation of the arcing with the onset of ELMs. From the simple expectation stated above one would expect that the light emission is oriented in the direction of the arc traces observed, i.e. perpendicular to the magnetic field direction. A subframe of 100 by 100 pixels integrated over a whole plasma discharge is shown in Fig 4a. Many events are found, which have the signature of arcs. But they are not orientated perpendicular to the magnetic field direction and each event is detected only in a single frame, i.e. there is no signature in the frame before and after this events. To exclude the influence of neutrons on the image guide and camera, the lens was covered to determine the effect by neutrons and indeed much less events were obtained for the same neutron rate.

To investigate the signature in the visible light further the identical camera was used in a laboratory evironment to record arcs on the same coatings as used AUG. The arc was ignited at a defined position [4] and moved in the presence of different magnetic fields. Even though the magnetic field was much weaker than in a tokamak, from the field dependence an extrapolation on the speed of the arc movement is possible. For magnetic fields up to $4 \mathrm{mT} \mathrm{a}$ 
linear dependence of the arc velocity on the magnetic field was obtained: $v_{\text {arc }}=30 \mathrm{~km} / \mathrm{s}^{*}$ $\mathrm{B}(\mathrm{T})$ [4]. Therefore an arc is visible in a field of view of 30 by $30 \mathrm{~mm}$ for less than $1 \mu \mathrm{s}$. In the experiments at AUG an exposure time of $3 \mu$ s was used. However, for a frame size of 256 by 256 pixels the maximal frame rate is restricted by the read out time, resulting in a frame rate of $26845 \mathrm{fps}$ or a read out time of $35 \mu \mathrm{s}$, during which the camera is not collecting data. This means that the camera used was too slow to record more than one frame per arc and the recorded trace shows only a small fraction of the full movement.

Although macroscopically arc traces look almost continous, an arc is a highly non linear phenomenon. Independent high speed camera measurements show that the internal dynamic is on a temporal scale of some ns. Furtheron, they yield that an arc does not move continously, but jumps from one buring point to the next. This movement is fractal, i.e. on bigger scale it is orientated perpendicular to the magnetic field direction, but on a microscopic scale the movement can be significantly different. This phenomenon is shown at the right side of Fig 4. The arc consits out of a row of spots. They move perpendicular to the magnetic field direction, but the light emission of the single spots varies significantly from frame to frame. If the resolution is reduced, as for the measurements in AUG, one will observe a structure, which is not orientated perpendicular to the magnetic field direction. 


\section{Summary}

The tungsten erosion by arcs in AUG was estimated by analysis of a large set of tiles. The amount of arc traces varies toroidally on a centimetre scale and even on a single tile. An automatised trace detection algorithm allowed determining the eroded material on a tile. In total about $2 \mathrm{~g}$ of tungsten were eroded during the 2009 campaign, which is a factor of 10 less than the net tungsten eosion by physical sputtering. Local erosion by arcing can destroy the tungsten coating of the tiles. Arcs seem to produce a significant amount of droplets and flakes. About $0.3 \mathrm{~g}$ of tungsten dust is found in AUG. As the droplets are ejected with a speed up to $80 \mathrm{~m} / \mathrm{s}$, one may expect a higher penetration probability into the core plasma than that of tungsten atoms. Laboratory investigations using a fast camera confirm the identification of events as arcs at the baffle region of AUG.

\section{References}

[1] R.Neu et al., this conference

[2] V. Rohde et al., J. Nucl. Mater. 415 (2011) S46

[3] A.Herrmann et al., ., J. Nucl. Mater. 390-391 (2011) 747

[4] M.Laux et al., this conference

[5] M. Mayer et al., Physica Scripta T138 (2009) 014039

[6] M.Balden et al, to be submitted to Nucl.Fusion.

[7] N. Endstrasser et al., J. Nucl. Mater. 415 (2011) S1085

[8] N. Endstrasser et al., Physica Scripta T145 (2011) 014021 
[9] M. Balden et al., this conference

[10] Mc Cracken et al. , J.Nucl.Mat. 93\&94 (1980)

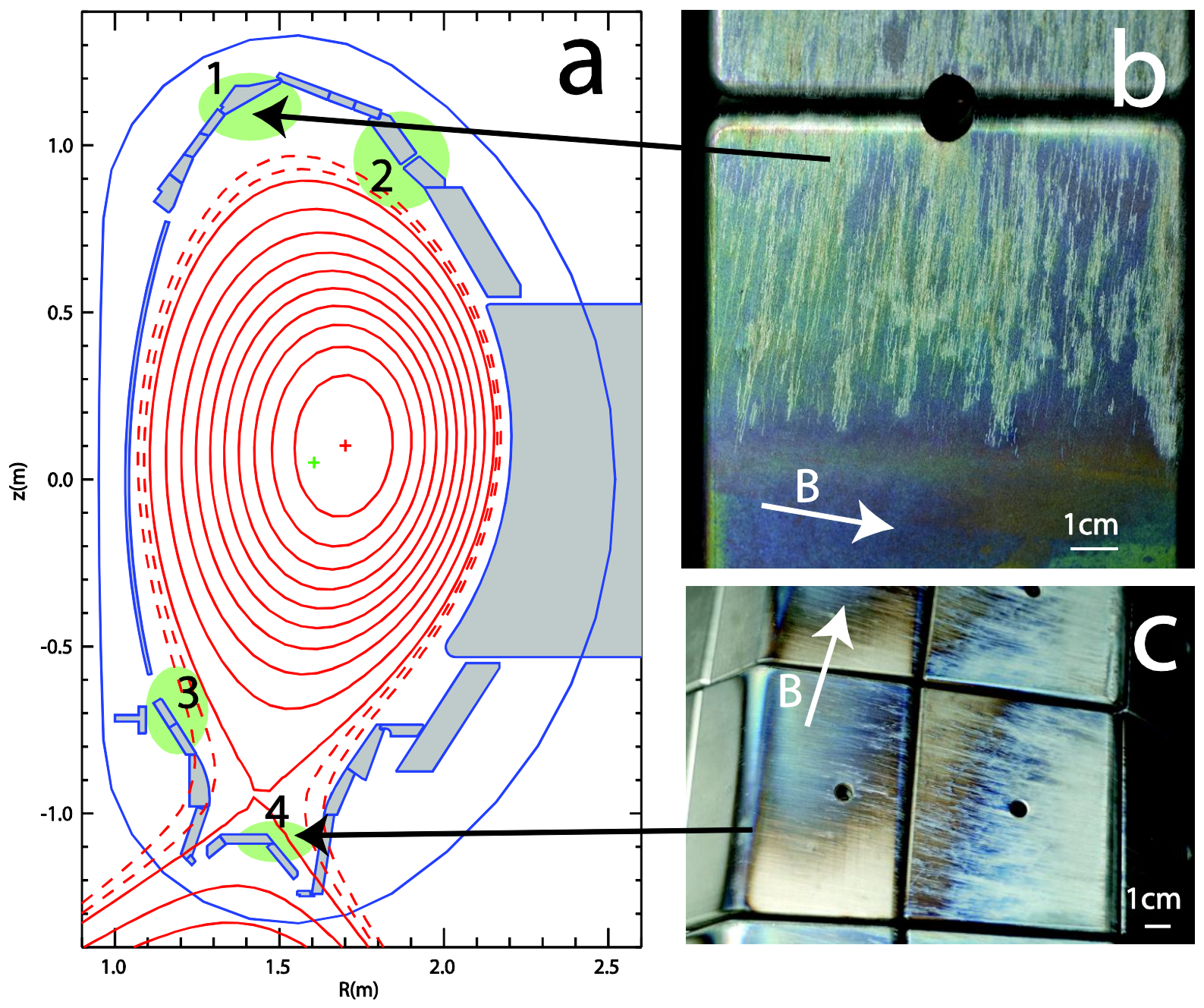

Fig.1 Cross section of AUG showing the locations where arc traces are observed: upper divertor close to the second x-point (1), upper divertor outside baffle region (2), lower inner divertor baffle region (3) and the divertor roof baffle (4). Inserts B and C show typical arc traces at the upper divertor region and the roof baffle. 


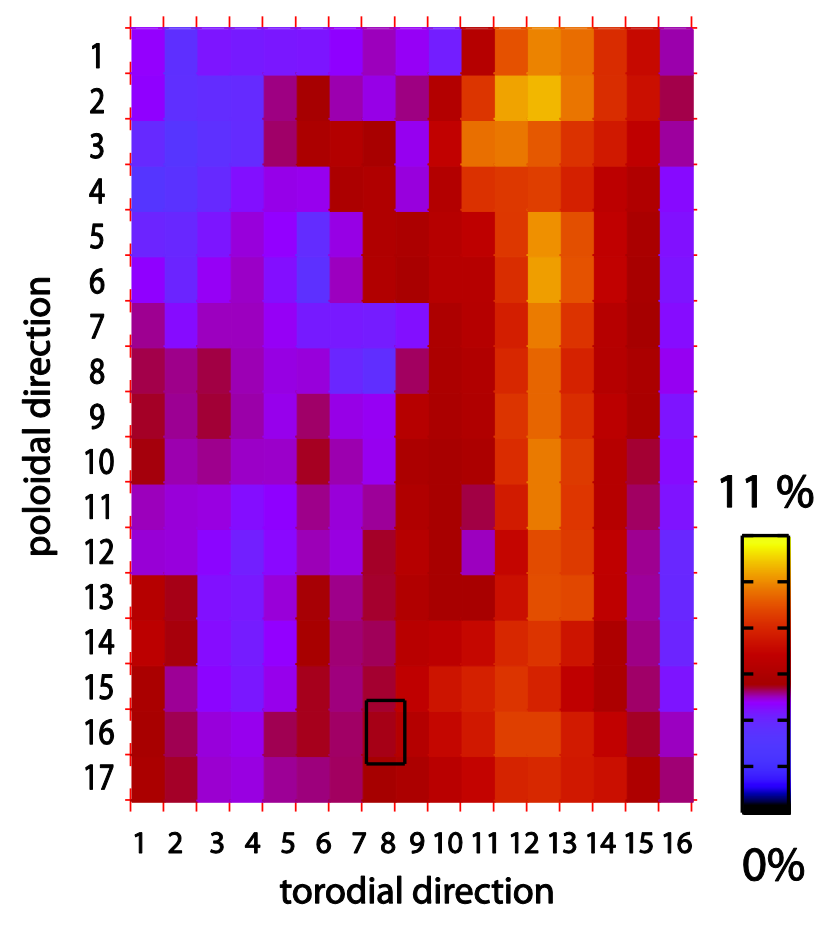

Fig.2 Results obtained by the arc detection algorithm for the tile 6B-2. 


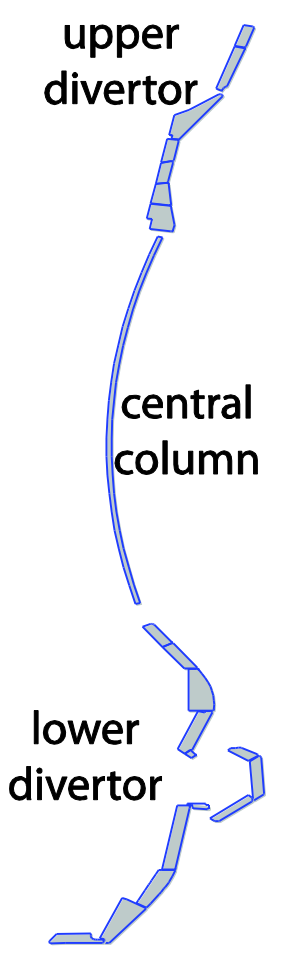

Surface fraction effected by arcing
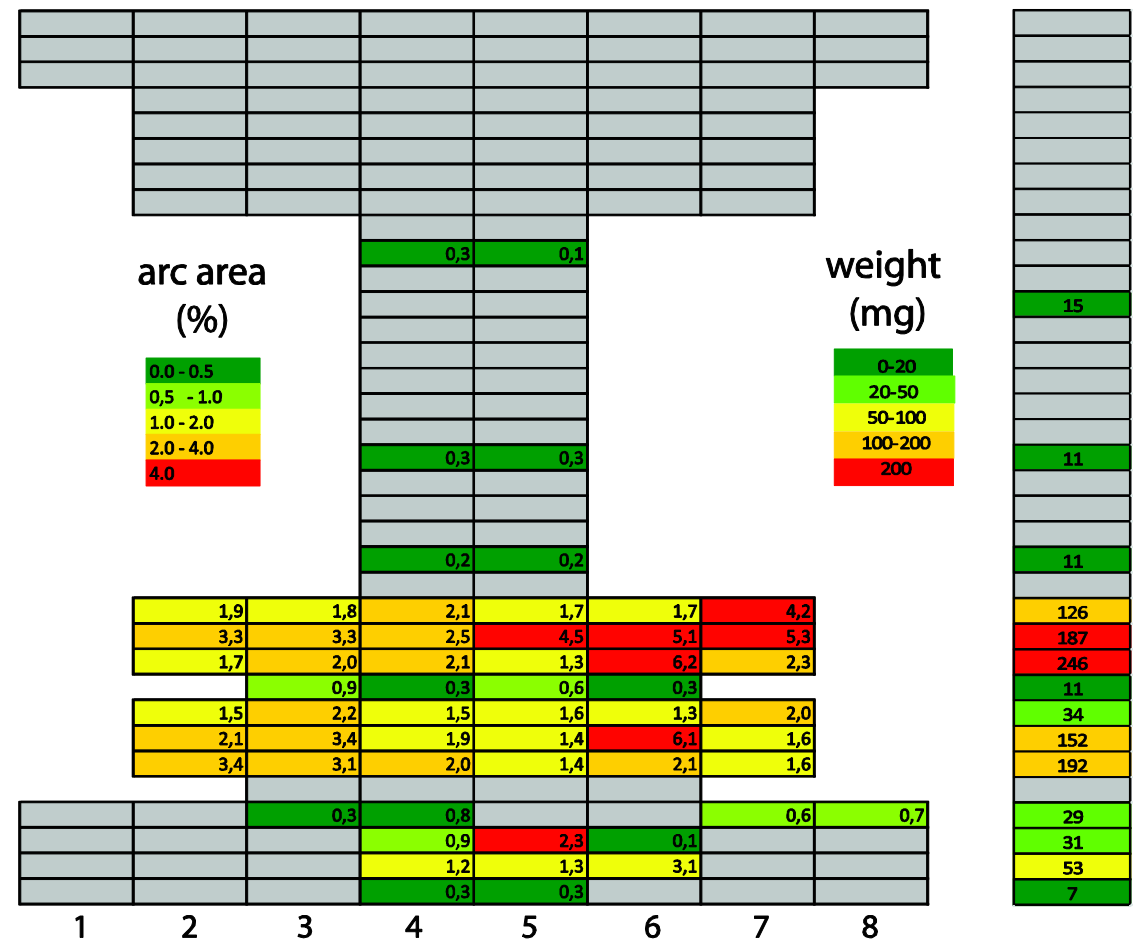

Fig.3 Erosion by arcing on the tiles of segment 11 from the upper divertor to the lower one. At the left side the shape of the tiles is shown as in Fig.1. In the middle the total fraction of the surface affected by arcing is shown. Tiles indicated in grey were not analysed. The derived erosion for the whole row of tiles is shown at the right side. 

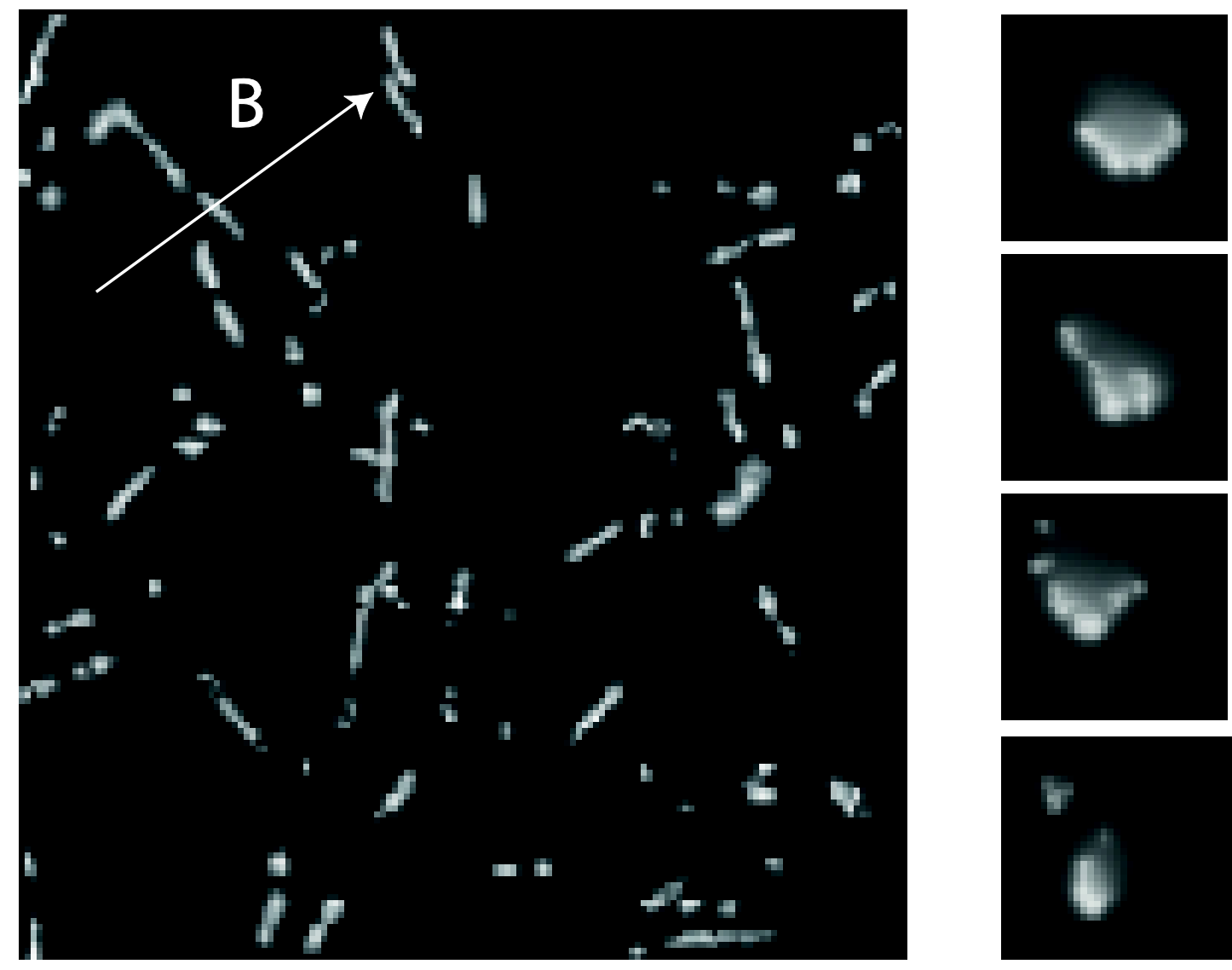

Fig.4 Signatures of arcs at the inner baffle region obtained by a fast camera integrated for $2 \mathrm{~s}$ during the flat top phase of discharge \# 25393. At the right side 4 subframes showing arcs in a laboratory environment are shown. 